\title{
Nucleus Accumbens Deep Brain Stimulation for Alcohol Addiction - Safety and Clinical Long-term Results of a Pilot Trial
}

Authors

Affiliations
U. J. Müller ${ }^{1}$, V. Sturm², J. Voges ${ }^{3}$, H.-J. Heinze ${ }^{4}$, I. Galazky ${ }^{4}$, L. Büntjen ${ }^{3}$, M. Heldmann ${ }^{4}$, T. Frodl ${ }^{1}$, J. Steiner ${ }^{1}$, B. Bogerts ${ }^{1}$

Department of Psychiatry, Otto-von-Guericke-University of Magdeburg, Magdeburg ${ }^{2}$ Department of Stereotaxy and Functional Neurosurgery, University of Cologne, Cologne

${ }^{3}$ Department of Stereotactic Neurosurgery, Otto-von-Guericke-University of Magdeburg, Magdeburg

${ }^{4}$ Department of Neurology, Otto-von-Guericke-University of Magdeburg, Magdeburg
Key words

alcohol addiction

- deep brain stimulation

nucleus accumbens

craving received $\quad 11.11 .2015$

revised 25.02.2016

accepted $\quad 29.02 .2016$

\section{Bibliography}

DOI http://dx.doi.org/

10.1055/s-0042-104507

Published online:

May 4, 2016

Pharmacopsychiatry 2016:

49: $170-173$

(c) Georg Thieme Verlag KG

Stuttgart · New York

ISSN 0176-3679

\section{Correspondence}

Dr. U. Müller

University Hospitals Magdeburg

Psychiatry

Leipzigerstr. 44

39120 Magdeburg

Germany

Ulf.Mueller@med.ovgu.de

\section{Abstract}

$\nabla$

We report on the long-term clinical outcome (up to 8 years) of 5 patients who received deep brain stimulation (DBS) of the nucleus accumbens to treat their long-lasting and treatment-resistant alcohol addiction. All patients reported a complete absence of craving for alcohol; 2 patients

\section{Introduction}

$\nabla$

In 2009, we published the first 3 cases of patients with severe treatment-resistant alcohol addiction who received bilateral deep brain stimulation (DBS) of the nucleus accumbens (NAc) to treat their disease [1]. In late 2008 and early 2009, two additional patients received DBS at our center as part of a pilot trial. In the years following the publication, DBS as a novel treatment for addiction has been applied to other addictions such as cocaine and heroin addiction in humans [2-4]. In addition, an increasing number of studies has been published on the effects of NAc DBS on specific addictive behaviors in rodent models [5]. Interestingly, although only a few patients with addiction have been included in clinical trials worldwide so far, the simple idea of treating addiction with neuromodulation led to multiple considerations regarding ethical issues. Respective publications tried to propose guidelines for future research but also raised concerns about brain stimulation for 'behavioral' disorders (sometimes even questioning the neurobiological aspects of addiction) or simply disapproved of the idea of treating addiction with brain stimulation [6-12].

This article describes the results and long-term experiences of 5 patients who received DBS to treat their severe and treatment-resistant addiction. While the experience is mainly clinical and no additional randomized scientific data was fur- remained abstinent for many years and 3 patients showed a marked reduction of alcohol consumption. No severe or long-standing side effects occurred. Therefore, DBS could be a promising, novel treatment option for severe alcohol addiction, but larger clinical trials are needed to further investigate the efficacy of DBS in addiction.

ther obtained, we still believe it is of importance to openly publish the long-term outcome of these patients ( $\odot$ Table 1 ).

\section{Patients and Methods}

$\nabla$

In addition to the 3 patients described before (patients 1-3 of the 2009 publication), 2 additional patients (patients 4 and 5) received bilateral DBS of NAc according to the protocol previously published [1].

In brief, inclusion criteria were as follows: male, age 25-60, inpatient detoxification followed by a minimum 2-week period of abstinence prior to surgery, a minimum 10-year history of alcoholism and at least 2 long-term rehabilitation treatments of at least 6 months in total as well as unsuccessful therapy with at least one of the anticraving medications (acamprosate, naltrexone) or disulfiram. Exclusion criteria were co-morbid mental retardation, withdrawal seizures during pharmacological detoxification, antisocial personality, visible brain damage in a CT or MRI scan as well as alcohol-related personality deprivation and abuse of other psychotropic substances with the exception of nicotine. Pre- and post-surgical assessment in the initial years included Symptom Checklist-90 (SCL), clinical psychopathology, obsessive-compulsive drinking scale (OCDS), and Alcohol Urge Questionnaire (AUQ). Surgery was performed at the Department of Stereotactic 
Neurosurgery and clinical examinations were performed at the Departments of Psychiatry and Neurology, all at the University Hospital of Magdeburg.

\section{Case vignettes of previously unpublished patients}

Patient 4 was born in 1957 and received DBS in December of 2008. He started consuming alcohol during his compulsory military service when he was 20 years old. By the time he was 25 , his son was born with mental and physical disabilities and the patient started to consume alcohol almost daily and often heavily, mainly to relieve stress. He recalls definitely being addicted by the time he was 30 but he first entered treatment at 40 and remained abstinent for 4 years. Following his $2^{\text {nd }}$ long-term rehabilitation treatment, he remained abstinent for 2-3 years. He was a trained mechanic and worked full time until the age of 40 , when he first lost his job due to alcohol. He stated that one of the main reasons why he managed to stay abstinent after his first and second rehabilitation treatments was the relatively low level of negative stress and the fact that he was able to find jobs again. However, after his relapse after the second rehabilitation treatment, he could no longer find work and has been unemployed ever since. Treatment with acamprosate was unsuccessful. His ADS score was 22; his AUQ score was 20.

Patient 5 was born in 1953 and received DBS in February 2009. Until 1990, he consumed alcohol socially. Following German reunification, he was unable to keep his former job as an electrician and started to work as a janitor, when he started to consume alcohol early during the day with a colleague. In 1991, he suffered acute pancreatitis and he was told for the first time that he was addicted to alcohol. He continued to consume alcohol and first entered treatment in 1996. However, he received only one detoxification treatment and relapsed soon after. By 2000 he had undergone inpatient detoxification yearly, but never managed to stay abstinent for more then 1-2 months after treatment. In 2000 he entered inpatient rehabilitation treatment for 16 weeks and stayed abstinent for over 2 years. By 2006 he had completed several detoxification treatments as well as outpatient rehabilitation and psychotherapy, but never stayed abstinent for more than 1-2 months after completing treatments. Since 2006, his daily alcohol consumption increased rapidly, sometimes exceeding $600 \mathrm{~g}$ of ethanol a day. More than once he was found unconscious and comatose, requiring ICU monitoring at our university hospital. His ADS score was 33 and his AUQ was 14.

\section{Safety and Clinical Long-term Results of All Five Patients \\ $\nabla$}

All patients were offered not only continuous care of their DBS device but also continuous psychiatric outpatient care at our university hospital covered by their compulsory health care insurance, thus care was free of charge for all.

\section{Patient one}

He remains abstinent since initiation of DBS in October 2007 without a relapse, thus being alcohol-free for almost 8 years. He has never reported any side effects or negative effects due to the DBS device and received a rechargeable DBS generator in 2012. He works full-time as an artisan and often has to travel away from his hometown for weeks. He reports a continuous and total absence of craving for alcohol following DBS.
Table 1 Patient description.

\begin{tabular}{|lccccc|}
\hline & $\begin{array}{c}\text { Age at time } \\
\text { of DBS OP }\end{array}$ & $\begin{array}{c}\text { Years } \\
\text { addicted }\end{array}$ & $\begin{array}{c}\text { ADS score } \\
\text { before DBS }\end{array}$ & $\begin{array}{c}\text { AUQ Score } \\
\text { before DBS }\end{array}$ & $\begin{array}{c}\text { AUQ Score } \\
\text { after DBS }\end{array}$ \\
\hline Pat. 1 & 40 & 23 & 34 & 37 & 8 \\
\hline Pat. 2 & 35 & 17 & 41 & 29 & 8 \\
\hline Pat. 3 & 37 & 22 & 28 & 53 & 8 \\
\hline Pat. 4 & 51 & 21 & 22 & 20 & 8 \\
\hline Pat. 5 & 55 & 19 & 33 & 14 & 8 \\
\hline
\end{tabular}

\section{Patient two}

He remained abstinent since initiation of DBS in January 2008 without a relapse. After 2 years of abstinence he started to attend nursing school and became a licensed medical nurse in summer 2012. He moved away to a different city in another state and started to work full-time at an intermediate care unit at a hospital. He was lost to clinical follow-up at the end of 2014 when he was last contacted by phone, when he claimed to be abstinent, still working and feeling well. Based on clinical records, the generator must have run out of battery power but the patient did not contact our department to schedule an appointment and could not be reached as he changed his phone number and his address is not publicly listed.

\section{Patient three}

Since initiation of DBS in 2007, he continued to relapse as described before and relapses became more frequent during mid 2009, leading to another long-term inpatient rehabilitation treatment for 12 weeks in fall 2009. He managed to stay abstinent for about 2 months after and had multiple short relapses since spring 2010. As he recalled, due to DBS his craving for alcohol vanished completely and he always relapsed because of negative stress he could not handle otherwise. In fall 2010 he was imprisoned for almost 4 years due to revocation of an old suspended sentence. Reasons for revocation included multiple counts of using public transportation without a valid ticket and other misdemeanors added up. During his imprisonment, the battery ran out of power and he reported a recurrence of craving leading to a replacement of the generator. After discharge in spring 2014 he continued to stay abstinent for days to weeks but had multiple yet short relapses. While the benefit of DBS was increasingly questioned by our professional staff, the patient always emphasized that without DBS he would drink high amounts of alcohol daily due to craving. The patient died in early summer 2015 and was legally autopsied as he was a subject of research. While the results of the autopsy are not available due to legal reasons, it can be assumed that DBS itself had no influence on his death as legal investigations would have followed had the autopsy raised such a suspicion. Although cause of death remains unclear to us, it can be assumed that he died due to continuous consumption of alcohol.

\section{Patient four}

His clinical records and lab results indicated abstinence for over 12 months following initiation of DBS. He was first detoxified after 15 months and stated that he had only relapsed for a few days before, as he wanted to see what would happen. He had additional relapses with subsequent detoxifications after 17, 21, 26 and 31 months. Although he did not manage to stay completely abstinent after DBS started, he always stated that DBS helped him to not relapse continuously and relapses always happened due to stress, not craving. In contradiction to his lab 


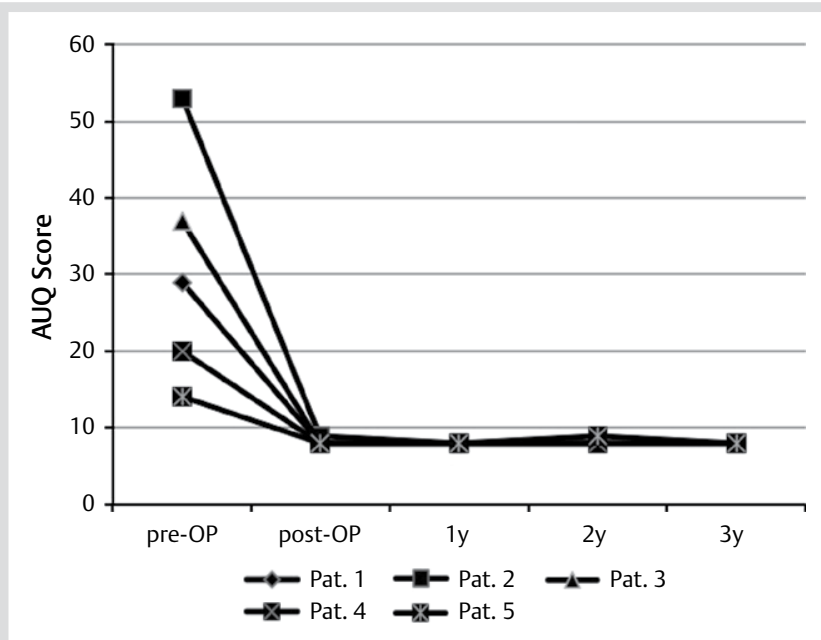

Fig. 1 shows the alcohol urge questionaire scores before and after surgery. A score of 8 is normal and indicates no craving. (Color figure available online only).

results, which always indicated a relapse for at least a week or two, he always insisted that his relapses were of a few days length only. After 36 months he suffered from a major depressive episode that was treated to remission with an SSRI and psychotherapy. However, he continued to relapse during the fourth year of DBS. As a main reason for relapse, he stated that he could not cope with the fact that his otherwise healthy and nonaddicted brother suddenly died a few years before and that he continued to live despite being addicted. While he was never suicidal and was offered inpatient therapy on numerous visits, he was lost to follow-up and we were informed that he was found dead at his home in late summer of 2012. Identical to patient 3, an autopsy was automatically ordered by a federal prosecutor, as cause of death was unknown, without further legal investigations into the DBS trial. Similarly, it can be assumed that he died due to continuous consumption of alcohol.

\section{Patient five}

He first relapsed for 2 days 20 months after DBS started and his lab results including CDT were normal. Since then he continues to have short relapses (up to 10 days) about every 2-3 months to "relieve" negative stress adding up to approximately 50 drinking days a year. He states that craving for alcohol had persistently disappeared and that the ratio of drinking days to abstinent days reversed after DBS was started. Before DBS, he was unable to be abstinent for 50 days in a year.

Clinically, he received outpatient psychotherapy treatment at our center 24 months after initiation of DBS for marital problems, feelings of guilt and depressive symptoms, as he did not manage to remain abstinent continuously and was thus unable to return to a substance-free life. Subsequently, after he and his wife finally separated in 2013, antidepressive medication (10 mg escitalopram) was started and depressive symptoms remitted.

\section{Summary Results and Side Effects \\ $\nabla$}

As outlined in the patient vignettes, all patients included had been severely addicted to alcohol and on average consumed at least $200 \mathrm{~g}$ ethanol a day. In addition, all patients had been highly resistant to existing treatment options and consumed high amounts of alcohol almost daily in the years before receiving DBS. All 5 patients reported a persistent disappearance of craving for alcohol ( $\bullet$ Fig. 1) and all patients acknowledged the fact that lack of craving was a main cause for abstinence or reduced alcohol consumption. Yet, although craving disappeared in all patients, not all patients were able to remain abstinent completely and 2 patients died after 4 and 8 years, respectively. With the exception of the previously reported hypomanic state of patient 2 directly after initiation of DBS, no adverse or serious adverse effects associated with DBS were observed in the followup period of up to 8 years. No patient reported any negative effects of DBS on their overall psychological well-being and no psychopathological symptoms were reported or observed during the follow-up period with the exception of depressive symptoms in patient 5 not associated with DBS. Stimulation parameters remained as previously published and no changes were made when patients relapsed. Surgeries for replacement of impulse generator were performed at our hospital as well and no side effects occurred during these procedures, which were financed by research grants. As all patients reported an absence of craving, stimulation parameters have not been changed throughout the course of treatment.

\section{Discussion}

The most striking single result of this pilot trial is the reported complete and persistent absence of craving for alcohol after initiation of NAc DBS. While AUQ scores differed in severity before DBS, AUQ dropped to normal after DBS in all patients. In addition to normal AUQ scores, patients also reported an absence of cueinduced craving in daily life, such as passing by a bar or seeing alcohol in a grocery store. In recent years, multiple fMRI and PET studies have shown that - by serving as an unconditioned stimulus - repeated intake of alcohol allows drug-related cues to become conditioned stimuli predictive of a drug response, thus leading to dopamine release and craving. Clinically, consumption of alcohol shifts from a goal directed towards habitual behavior [13]. Thus, the reported absence of craving for alcohol is in line with the hypothesis that DBS of the NAc may normalize or "free" the reward system of the brain, which has been "hijacked" by alcohol [14]. However, although DBS eliminated the craving for alcohol, it did not lead to complete abstinence in all patients treated.

In our patients, we used the common AUQ self-rating scale to measure craving. This, of course, measures only the conscious craving for alcohol, so patients have to be aware of it. Yet it has been postulated that automatic or habitual alcohol intake without conscious craving may also play an important part in relapse [15-17] and could explain why some patients relapsed although they no longer experienced craving. In addition, relapse can also be explained by negative reinforcement, which tends to become more relevant in the later course of addiction and could explain why the 2 youngest patients did not relapse, whereas the older patients, who had much lower craving levels on the AUQ [18,19], did.

All of the above-mentioned possible reasons for relapse are based on the acute and long-term psychotropic effects of alcohol leading to dysfunctional brain circuits. However, aside from its psychotropic effects on the function of brain circuits, alcohol also has neurotoxic potency, leading to irreversible structural damage of brain tissue $[20,21]$. Therefore, relapse might also be explained by structural brain damage of relapse-associated brain circuits, which is supported by recent studies showing that 
morphological abnormalities [22] and atrophy correlate with risk of relapse [23].

Considering the fact that response rates of available treatment interventions range between 30 to $85 \%[24,25]$ there clearly is a need for novel treatments in alcohol addiction. Keeping in mind that our patients were severely affected by their alcohol addiction and were non-responders to available treatment options before DBS was initiated, we believe that our results are very promising. Thus, larger double-blind and sham-controlled trials are needed to assess the impact of NAc DBS on craving and its clinical relevance in treating alcohol addiction. However, recent experience with DBS trials in cocaine and heroin addiction showed that DBS seems to be rather unattractive in affected individuals [3].

Aside from the clinical aspects, we believe that the experiences of patients with DBS are also noteworthy. All patients reported that their overall experience with DBS was very positive. They all reported a health benefit attributed to DBS and none of the patients reported side effects or negative consequences associated with DBS, which they would have accepted as a compromise. Interestingly, DBS did not suppress the reward system entirely or lead to lack of pleasurable feelings in normal or daily situations but rather enabled patients to experience pleasurable or rewarding feelings again.

As mentioned in the case vignettes, 2 patients died after 4 years and 8 years of DBS, respectively. While death of patients is always tragic, the death of patients actively enrolled in clinical research is always alarming initially and thorough investigations into cause of death are needed to ensure that no harm was done because of the research intervention. Keeping in mind that mortality is dramatically increased in alcohol addiction $[26,27]$, the fact that our patients died years after initiation of DBS makes it very unlikely that DBS is a causal link in their deaths. This is supported by the fact that no formal or judicial investigation was initiated following the autopsies ordered by the federal prosecutor.

In summary, the long-term clinical outcome of the 5 patients who received DBS to treat their long-lasting and treatmentresistant alcohol addiction seems promising and justifies further clinical investigations.

\section{Important note}

$\nabla$

During the process of proofreading the accepted manuscript, we were finally able to obtain the legal autopsy reports of both deceased patients. In both cases no causal relationship between DBS and death was found or suspected. Both patients died most likely due to severe alcohol withdrawal. However, as both bodies were found $>24 \mathrm{~h}$ after time of death, no definitely cause of death could be determined.

\section{Conflict of Interest}

$\nabla$

The authors declare no conflict of interest.

\section{References}

1 Müller UJ, Sturm V, Voges J et al. Successful treatment of chronic resistant alcoholism by deep brain stimulation of nucleus accumbens: first experience with three cases. Pharmacopsychiatry 2009; 288-291 doi:10.1055/s-0029-1233489

2 Gonçalves-Ferreira A, do Couto FS, Campos AR et al. Deep brain stimulation for refractory cocaine dependence. Biol Psychiatry 2015, doi:10.1016/j.biopsych.2015.06.023
3 Luigjes J, van den Brink W, Schuurman PR et al. Is deep brain stimulation a treatment option for addiction? Addiction 2015; 547-548 doi:10.1111/add.12773

4 Kuhn J, Möller M, Treppmann JF et al. Deep brain stimulation of the nucleus accumbens and its usefulness in severe opioid addiction. Mol Psychiatry 2014; 145-146 doi:10.1038/mp.2012.196

5 Pierce RC, Vassoler FM. Deep brain stimulation for the treatment of addiction: basic and clinical studies and potential mechanisms of action. Psychopharmacology 2013; 487-491 doi:10.1007/s00213-013-3214-6

6 Muller UJ, Voges J, Steiner J et al. Deep brain stimulation of the nucleus accumbens for the treatment of addiction. Ann N York Acad Sci 2013; 119-128 doi:10.1111/j.1749-6632.2012.06834.X

7 Muller UJ, Bogerts B, Voges J et al. Deep brain stimulation in psychiatry: ethical aspects. Psychiatr Prax 2014;38-43 doi:10.1055/s-0034-1370015

8 Kuhn J, Möller M, Müller U et al. Deep brain stimulation for the treatment of addiction. Addiction 2011; 1536-1537 author reply 15361537 doi:10.1111/j.1360-0443.2011.03452.x

9 Carter A, Bell E, Racine E et al. Ethical issues raised by proposals to treat addiction using deep brain stimulation. Neuroethics 2010; 129-142 doi:10.1007/s12152-010-9091-3

10 Pisapia JM, Halpern $\mathrm{CH}$, Muller UJ et al. Ethical considerations in deep brain stimulation for the treatment of addiction and overeating associated with obesity. AJOB Neuroscience. 2013 35-46 doi:10.1080/21 507740.2013.770420

11 Woopen C, Pauls KAM, Koy A et al. Early application of deep brain stimulation: clinical and ethical aspects. Prog Neurobiol 2013; 74-88 doi:10.1016/j.pneurobio.2013.04.002

12 Carter $A$, Hall W. Proposals to trial deep brain stimulation to treat addiction are premature. Addiction 2011; 235-237 doi:10.1111/ j.1360-0443.2010.03245.x

13 Everitt BJ, Robbins TW. Neural systems of reinforcement for drug addiction: from actions to habits to compulsion. Nat Neurosci 2005; 1481-1489 doi:10.1038/nn1579

14 Jasinska AJ, Stein EA, Kaiser J et al. Factors modulating neural reactivity to drug cues in addiction: a survey of human neuroimaging studies. Neurosci Biobehav Rev 2014; 1-16 doi:10.1016/j.neubiorev.2013.10.013

15 Tiffany ST. A cognitive model of drug urges and drug-use behavior: role of automatic and nonautomatic processes. Psychol Rev 1990; 147-168 doi:10.1037/0033-295X.97.2.147

16 Tiffany ST, Wray JM. The clinical significance of drug craving. Ann N Y Acad Sci 2012; 1-17 doi:10.1111/j.1749-6632.2011.06298.x

17 Garbusow M, Sebold M, Beck A et al. Too difficult to stop: mechanisms facilitating relapse in alcohol dependence. Neuropsychobiology 2014; 103-110 doi:10.1159/000362838

18 Wise RA, Koob GF. The development and maintenance of drug addiction. Neuropsychopharmacology 2014; 254-262 doi:10.1038/ npp.2013.261

19 Koob GF. The dark side of emotion: the addiction perspective. Eur J Pharmacol 2015; 73-87 doi:10.1016/j.ejphar.2014.11.044

20 la Monte de SM, Kril JJ. Human alcohol-related neuropathology. Acta Neuropathol 2014; 71-90 doi:10.1007/s00401-013-1233-3

21 Buhler M, Mann K. Alcohol and the human brain: a systematic review of different neuroimaging methods. Alcohol Clin Exp Res 2011; 17711793 doi:10.1111/j.1530-0277.2011.01540.x

22 Durazzo TC, Tosun D, Buckley S et al. Cortical thickness, surface area and volume of the brain reward system in alcohol dependence: relationships to relapse and extended abstinence. Alcohol Clin Exp Res 2011; 1187-1200 doi:10.1111/j.1530-0277.2011.01452.x

23 Beck A, Wüstenberg T, Genauck A et al. Effect of brain structure, brain function, and brain connectivity on relapse in alcohol-dependent patients. Arch Gen Psychiatry 21012: 842-852 doi:10.1001/archgenpsychiatry.2011.2026

24 Naqvi NH, Morgenstern J. Cognitive neuroscience approaches to understanding behavior change in alcohol use disorder treatments. Alcohol Res 2015; 37: 29-38

25 Maisel NC, Blodgett JC, Wilbourne PL et al. Meta-analysis of naltrexone and acamprosate for treating alcohol use disorders: when are these medications most helpful? Addiction 2013; 275-293 doi:10.1111/ j.1360-0443.2012.04054.x

26 Roerecke $M$, Rehm J. Alcohol use disorders and mortality: a systematic review and meta-analysis. Addiction 2013; 1562-1578 doi:10.1111/ add. 12231

27 Roerecke $M$, Rehm J. Cause-specific mortality risk in alcohol use disorder treatment patients: a systematic review and meta-analysis. Int J Epidemiol 2014; 906-919 doi:10.1093/ije/dyu018 